\title{
Mise en évidence de l'adsorption de l'isopentane dans les pores de la zéolithe $5 \mathrm{~A}$
}

\author{
P. Magnoux ${ }^{1}$, Y. Boucheffa ${ }^{1}$, M. G uisnet ${ }^{1}$, G. Joly ${ }^{1}$ et S. Jullian² \\ 1 UMR 6503, Laboratoire de catalyse en chimie organique, 40, avenue du Recteur-Pineau, 86022 Poitiers Cedex - France \\ 2 Institut français du pétrole, 1 et 4 , avenue de Bois-Préau, 92852 Rueil-M almaison Cedex - France \\ e-mail : patrick.magnoux@campus.univ-poitiers.fr - sophie.jullian@ifp.fr
}

\begin{abstract}
Résumé - Sur zéolithe 5A, l'adsorption de l'isopentane et du $n$-pentane a été suivie en microbalance dans une gamme de températures variant de 50 à $420^{\circ} \mathrm{C}$ et pour une pression d'alcanes de $97 \mathrm{kPa}$. L'analyse de la phase adsorbée montre clairement que l'isopentane entre dans les pores de l'adsorbant et reste bloqué dans les cages $\alpha$ de la zéolithe, et ceci quelle que soit la température. À basse température $\left(T=50^{\circ} \mathrm{C}\right)$, chaque cage $\alpha$ peut être occupée par trois molécules d'isopentane. Les molécules d'isopentane peuvent facilement être désorbées par un traitement sous vide primaire à la température de l'adsorption. À haute température $\left(T \geq 250^{\circ} \mathrm{C}\right)$, une partie de l'isopentane bloqué dans les pores est transformé en coke (oligomères et composés mono- et biaromatiques) par réactions successives sur les sites acides de la zéolithe (craquage, oligomérisation, cyclisation et transfert d'hydrogène). Ces molécules bloquées dans les cages $\alpha$ de la zéolithe limitent la désorption de l'isopentane.
\end{abstract}

Mots-clés : zéolithe 5A, adsorption, isopentane, coke.

\begin{abstract}
Evidence for Isopentane Adsorption in the Pores of 5A Zeolite - Adsorption of isopentane and $\mathrm{n}$-pentane on $5 \mathrm{~A}$ zeolite was investigated using a microbalance at temperatures between 50 and $420^{\circ} \mathrm{C}\left(P_{\text {alkanes }}=97 \mathrm{kPa}\right)$. Analysis of the adsorbed phase shows that, despite the well-known geometric selectivity of $5 \mathrm{~A}$ zeolite, isopentane enters the pores and remains trapped in the $\alpha$ cages. At saturation at $50^{\circ} \mathrm{C}$, all the $\alpha$ cages of the $5 \mathrm{~A}$ zeolite are occupied by three isopentane molecules. Isopentane molecules are completely removed from the zeolite pores by an adequate vacuum treatment at the adsorption temperature. At high temperature $\left(T \geq 250^{\circ} \mathrm{C}\right)$, trapped isopentane molecules are transformed into "coke" (oligomers and aromatic compounds) by successive reactions (cracking, polymerisation, cyclisation and hydrogen transfer) on the acid sites of 5A zeolite. The "coke" molecules, blocked inside the $\alpha$ cages, limit the desorption of isopentane (and $\mathrm{n}$-pentane).
\end{abstract}

Keywords: $5 A$ zéolite, adsorption, isopentane, coke. 


\section{INTRODUCTION}

Le fractionnement initial des pétroles bruts par distillation fournit une coupe essence à faible indice d'octane. Celui de l'essence légère $\left(\mathrm{C}_{5}-\mathrm{C}_{6}\right)$ varie entre 60 et 80 et celui de l'essence lourde $\left(\mathrm{C}_{7}-\mathrm{C}_{10}\right)$ entre 30 et 50 . Comme les moteurs à allumage commandé exigent des indices d'octane relativement élevés (95-98) pour le supercarburant, les coupes essences doivent être améliorées, soit par incorporation d'additifs comme le plomb tétraéthyle ; soit en transformant les hydrocarbures à faible indice d'octane en hydrocarbures à indice d'octane plus élevé.

Le plomb tétraéthyle ajouté aux essences jusque dans les années 80 permettait d'en augmenter l'indice d'octane. Les normes environnementales d'une part, et l'effet néfaste des additifs à base de plomb sur la durée de vie des pots catalytiques qui équipent les nouvelles voitures d'autre part, ont conduit les raffineurs à produire de l'essence à plus haut indice d'octane. Pour pallier la perte d'octane provoquée par la suppression du plomb tétraéthyle, divers procédés permettant d'obtenir des bases essences à haut indice d'octane se développent, tels que :

- l'alkylation isobutane-butène ;

- la synthèse de bases oxygénées (MTBE, TAME, etc.) ;

- l'isomérisation des coupes $\mathrm{C}_{5} / \mathrm{C}_{6}$.

L'isomérisation permet de convertir une fraction des paraffines linéaires à bas indice d'octane en paraffines branchées possédant un meilleur indice d'octane. Toutefois, la conversion en isomères est limitée par la thermodynamique et favorisée à basse température. Même en utilisant un catalyseur d'isomérisation très actif, toutes les $n$-paraffines ne sont pas converties et l'indice d'octane du mélange est limité à 82-84 [1]. Le recyclage des $n$-paraffines non transformées jusqu'à extinction permet d'obtenir des gains d'octane supplémentaires.

Le recyclage des paraffines normales vers l'isomérisation implique la séparation des isomères branchés. L'utilisation de tamis moléculaire, comme la zéolithe $5 \mathrm{~A}$, permet la séparation des isoalcanes. La technique de séparation par tamis moléculaire repose sur la faculté de la zéolithe utilisée d'adsorber sélectivement dans ses pores les paraffines normales du fait de leurs diamètres cinétiques et de laisser passer les isoparaffines à cause de leurs diamètres cinétiques légèrement plus grands. L'étape d'adsorption est suivie d'une étape de désorption pour la récupération des paraffines normales. De nombreux procédés d'adsorption utilisant la zéolithe 5A, comme les procédés Molex de l'UOP [2] ou IPSORB de l'IFP [3] ont été développés.

Toutefois, comme dans tout procédé utilisant des zéolithes, un problème majeur, qui se produit au cours de la séparation, est la désactivation de la zéolithe $5 \mathrm{~A}$ par perte de sa capacité d'adsorption. Cette désactivation est généralement due à la formation et à la rétention de composés carbonés, souvent appelés coke, dans les pores de la zéolithe. Le dépôt carboné formé sur la zéolithe 5A au cours de la séparation des $n$-alcanes et isoalcanes peut résulter de la transformation d'alcènes [4-7], qui sont présents à l'état de traces dans la charge ou qui résultent d'un craquage des alcanes ou des isoalcanes.

Une autre cause de désactivation de la zéolithe $5 \mathrm{~A}$ peut être l'adsorption et le piégeage des isoalcanes dans les pores de la zéolithe.

L'objectif de ce travail a donc été de montrer que des isoalcanes, tels que l'isopentane, pouvaient entrer dans les pores de la zéolithe $5 \mathrm{~A}$ et y rester piégés dans certaines conditions opératoires.

\section{PARTIE EXPÉRIMENTAL}

\subsection{Zéolithe}

La zéolithe $5 \mathrm{~A}\left(\mathrm{Na}_{3,1} \mathrm{Ca}_{4,45} \mathrm{Al}_{12} \mathrm{Si}_{12} \mathrm{O}_{48}\right)$ utilisée dans cette étude a été fournie par la société Procatalyse. L'analyse par microscopie électronique montre la présence de cristaux de forme cubique de 1 à $2 \mu \mathrm{m}$. Cette zéolithe possède un volume poreux accessible à l'azote de $0,260 \mathrm{~cm}^{3} \mathrm{~g}^{-1}$, et au $n$ hexane de $0,230 \mathrm{~cm}^{3} \mathrm{~g}^{-1}$. La thermodésorption d'ammoniac indique l'existence d'une certaine acidité, cette dernière pouvant être reliée à la présence de sites acides de Lewis $\left(\mathrm{Ca}^{2+}\right)$ ou de sites acides protoniques résultant d'une hydrolyse partielle des cations $\mathrm{Ca}^{2+}[8,9]$.

\subsection{Adsorption - Désorption}

Les mesures d'adsorption du $n$-pentane et de l'isopentane sur la zéolithe $5 \mathrm{~A}$ ont été réalisées dans une gamme de températures allant de 50 à $420^{\circ} \mathrm{C}$ à l'aide d'une microbalance de type Sartorius 4433. L'échantillon $(0,085 \mathrm{~g})$ est calciné sous air à $500^{\circ} \mathrm{C}$ pendant trois heures. La perte de masse de l'échantillon au cours de ces traitements étant d'environ $30 \%$, la masse résiduelle de l'échantillon est toujours voisine de 0,060 g. Après séchage sur tamis moléculaire $3 \mathrm{~A}$, le $n$-pentane (pureté $=99,4 \%$ ) et l'isopentane (pureté $=99,8 \%$ ) sont introduits à $97 \mathrm{kPa}$. Afin d'éviter toute condensation, l'ensemble de l'installation est maintenu à $40^{\circ} \mathrm{C}$. Après chaque expérience, afin de définir la vitesse de désorption de l'alcane et de déterminer la quantité de matière non désorbable (coke), l'échantillon est traité sous vide (1 $\mathrm{Pa})$ à la température de l'adsorption, jusqu'à obtention d'une masse constante.

\subsection{Analyse de la phase adsorbée}

Pour analyser la phase adsorbée, plusieurs échantillons ont été préparés in situ en microbalance afin d'obtenir environ $1 \mathrm{~g}$ de zéolithe. Les composés ont été analysés en utilisant la 

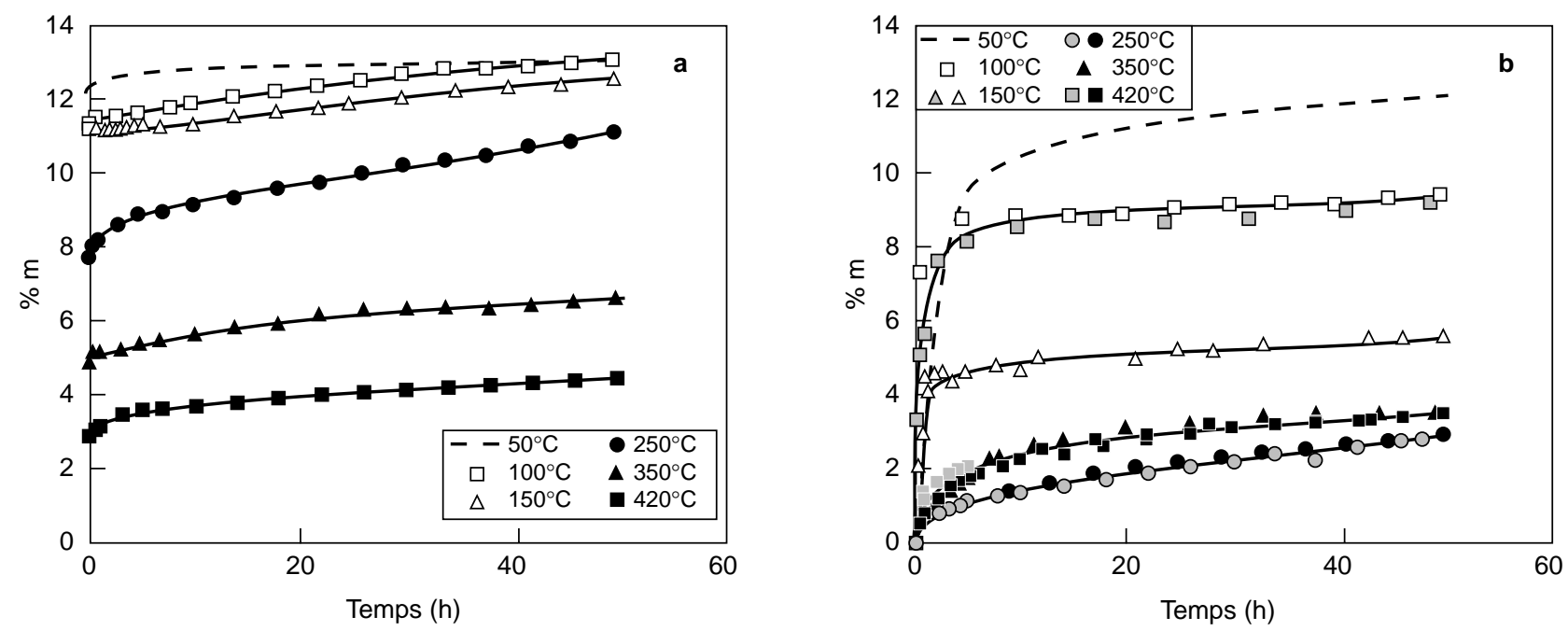

Figure 1

Prise de masse sur la zéolithe 5A (\% m) en fonction du temps pour diverses températures. (a) $n$-pentane, (b) isopentane.

Weight increase of 5 A zeolite versus time for various temperatures. (a) $\mathrm{n}$-pentane, (b) isopentane.

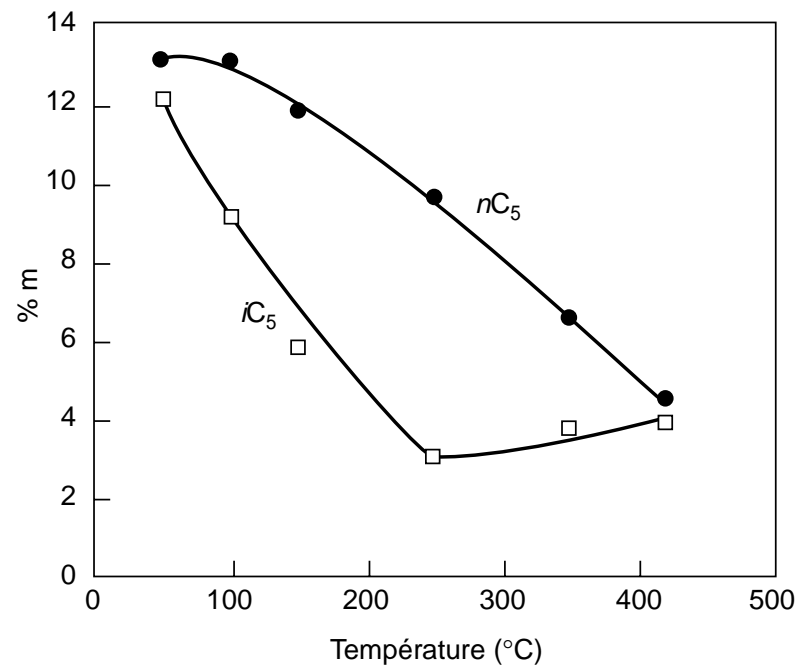

Figure 2

Prise de masse sur la zéolithe $5 \mathrm{~A}(\% \mathrm{~m})$ après 50 heures de contact avec le $n$-pentane et l'isopentane en fonction de la température.

Weight of the 5 A zeolithe after 50 hours contact with $\mathrm{n}$ pentane and isopentane versus operating temperature.

technique développée au laboratoire pour l'analyse de coke [10]. La zéolithe est solubilisée à température ambiante dans de l'acide fluorhydrique à $40 \%$ afin de libérer les molécules organique piégées dans ses pores. Nous avions préalablement vérifié que cette technique n'affectait en rien la nature de ces composé organiques [11]. Dans ce cas précis, le gaz libéré durant cette opération est récupéré puis analysé par chromatographie en phase gazeuse (CPG) à l'aide d'une colonne capillaire (Plot - $\mathrm{Al}_{2} \mathrm{O}_{3} / \mathrm{KC} 1$ ) tandis que les autres composés sont solubilisés dans du chlorure de méthylène et analysés par CPG (colonne capillaire DB5) et par couplage chromatographie en phase gazeuse-spectrométrie de masse (CPG-SM).

\section{RÉSULTATS}

\subsection{Cinétique d'adsorption du n-pentane et de l'isopentane}

Sur zéolithe 5A, l'adsorption du $n$ - et de l'isopentane a été suivie en fonction du temps, à $97 \mathrm{kPa}$ et pour des températures variant entre 50 et $420^{\circ} \mathrm{C}$ (fig. 1). Quel que soit l'alcane utilisé, la prise de masse $(\% \mathrm{~m})$ est relativement rapide durant les premières minutes puis diminue par la suite, pour atteindre un plateau après environ 20 heures. À saturation (après 50 heures), la prise de masse peut atteindre jusqu'à 12 et $13 \%$ à $50^{\circ} \mathrm{C}$, respectivement pour le $n$-pentane et l'isopentane.

Pour le $n$-pentane, la prise de masse à saturation décroît quasi linéairement avec la température, passant de 13 à 4,5\% entre 50 et $420^{\circ} \mathrm{C}$ (fig. 2). Cette décroissance de l'adsorption du $n$-pentane sur zéolithe $5 \mathrm{~A}$ corrobore les résultats obtenus par Bin et al. [12] sur adsorbant 5A, ainsi que ceux reportés par Silva et Rodrigues [13]. 


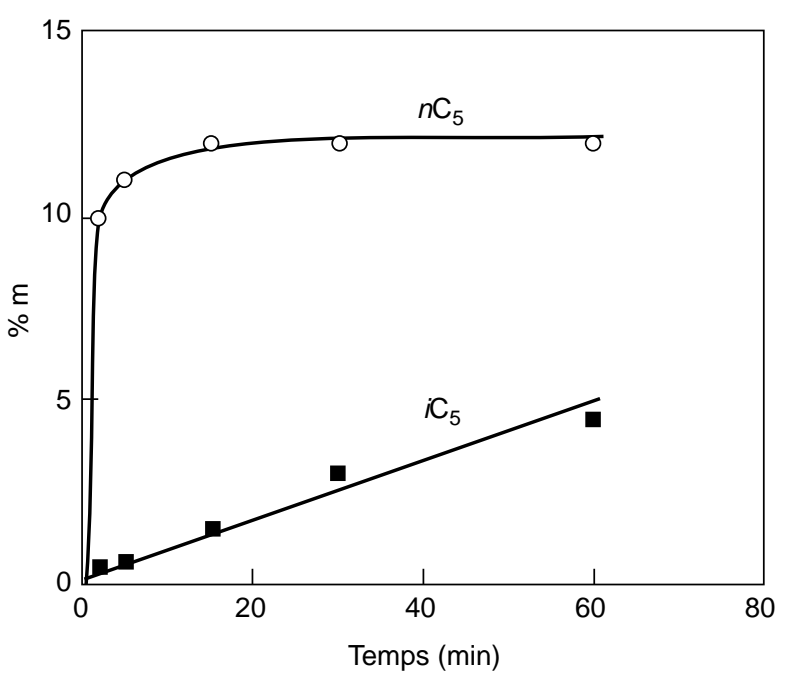

Figure 3

Prise de masse initiale du $n$-pentane et de l'isopentane à $50^{\circ} \mathrm{C}$. Initial weight increase for $\mathrm{n}$-pentane and isopentane at $50^{\circ} \mathrm{C}$.

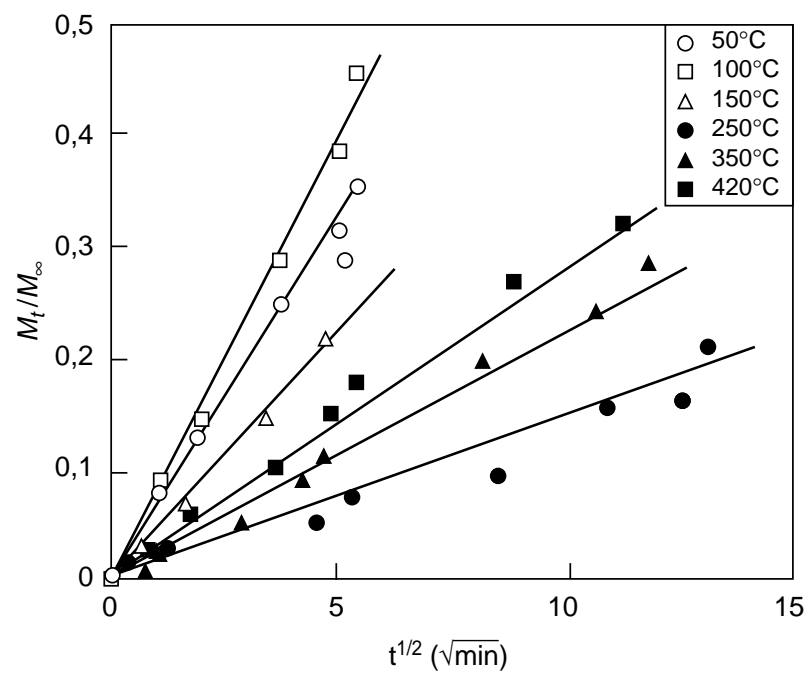

Figure 4

Cinétiques de diffusion de l'isopentane dans la zéolithe 5A pour diverses températures.

Kinetics of diffusion of isopentane over 5 A zeolite for various temperatures.
Dans le cas de l'isopentane, la quantité adsorbée à saturation décroît de 12 à $3 \%$ lorsque la température augmente de 50 à $250^{\circ} \mathrm{C}$, puis augmente légèrement entre 250 et $420^{\circ} \mathrm{C}$ (fig. 2). À $50^{\circ} \mathrm{C}$, la prise de masse après 50 heures d'adsorption est quasiment indépendante de l'alcane considéré, alors qu'entre 100 et $250^{\circ} \mathrm{C}$, elle est plus significative avec le $n$-pentane. À plus haute température, cette différence s'atténue et les prises de masse pour le $n$ - et l'isopentane sont quasiment identiques à $420^{\circ} \mathrm{C}$.

$\mathrm{Si}$, à faible température, les prises de masse à saturation sont voisines entre $n$ - et isopentane, l'adsorption initiale du $n$ pentane est nettement plus rapide (fig. 3). Malgré l'extrême rapidité de l'adsorption de la paraffine linéaire, nous avons estimé, pour chaque température, les vitesses initiales d'adsorption (calculées après 15 secondes) de chacune des paraffines (tableau 1). Ainsi, jusqu'à $250^{\circ} \mathrm{C}$, l'adsorption du $n$-pentane est environ 50 fois plus rapide que celle de l'isopentane, alors qu'à plus haute température $\left(350-420^{\circ} \mathrm{C}\right)$, la différence d'adsorption des deux molécules s'atténue.

\section{TABLEAU 1}

Vitesse initiale d'adsorption $\left(10^{-3} \mathrm{~mol} \mathrm{~h}^{-1} \mathrm{~g}^{-1}\right)$

de l'isopentane et du $n$-pentane sur la zéolithe $5 \mathrm{~A}$

Initial adsorption rates $\left(10^{-3} \mathrm{~mol} \mathrm{~h}^{-1} \mathrm{~g}^{-1}\right)$

of isopentane and $\mathrm{n}$-pentane over 5 A zeolite

\begin{tabular}{c|c|c|c|c|c|c}
\hline$T\left({ }^{\circ} \mathrm{C}\right)$ & 50 & 100 & 150 & 250 & 350 & 420 \\
\hline$i \mathrm{C}_{5}$ & 7 & 7 & 5 & 4 & 20 & 15 \\
\hline$n \mathrm{C}_{5}$ & 350 & 320 & 250 & 220 & 100 & 80 \\
\hline
\end{tabular}

Nous avons essayé également d'estimer la diffusion apparente de l'isopentane et du $n$-pentane dans la zéolithe 5A en fonction de la température. Pour ceci, nous avons utilisé la loi de Fick quand le temps tend vers zéro [14] :

$$
M_{t} / M_{\infty}=6 / \sqrt{\pi\left(D / r_{0}^{2} t\right)^{1 / 2}}
$$

où $M_{t}$ et $M_{\infty}$ sont les quantités adsorbées à l'instant $t$ et à l'infini, $D$ le coefficient de diffusion et $r_{0}$ le rayon des cristallites de la zéolithe.

Les courbes qui représentent le rapport $M_{t} / M_{\infty}$ en fonction de la racine du temps nous permettent d'estimer les valeurs de ces diffusions apparentes $D / r^{2}{ }_{0}$ à partir des pentes à l'origine. À titre d'exemple, nous donnons l'une de ces courbes pour l'isopentane en figure 4 . Les valeurs de diffusion apparente de l'iso- et du $n$-pentane sont données dans le tableau 2 en fonction de la température.

Pour l'isopentane, les valeurs de diffusion apparente sont entre 1000 et 10000 fois plus faibles que celles du $n$-pentane. Dans le cas du $n$-pentane, la diffusion augmente avec la température jusqu'à $150^{\circ} \mathrm{C}$ puis diminue par la suite. En revanche, avec l'isopentane, la diffusion de cet alcane augmente normalement jusqu'à $100^{\circ} \mathrm{C}$ pour ensuite diminuer quand la température augmente.

\subsection{Analyse de la phase adsorbée}

La phase adsorbée dans la zéolithe $5 \mathrm{~A}$ a été analysée après adsorption du $n$ - et de l'isopentane à 100,250 et $420^{\circ} \mathrm{C}$ pendant 50 heures. 
TABLEAU 2

Diffusion apparente de l'iso- et du $n$-pentane dans la zéolithe $5 \mathrm{~A}$ en fonction de la température

Influence of the temperature on the diffusivity of iso- and n-pentane for 5 A zeolite

\begin{tabular}{c|c|c|c|c|c|c}
\hline$T\left({ }^{\circ} \mathrm{C}\right)$ & 50 & 100 & 150 & 250 & 350 & 420 \\
\hline $\begin{array}{c}10^{-8} \cdot D / r_{0}^{2} \\
\left(\mathrm{~s}^{-1}\right)\end{array}$ & 10,3 & 12,5 & 8,7 & 0,85 & 1,1 & 1,6 \\
\hline $\mathrm{C}_{5}$ & 0,7 & 1,3 & 1,4 & 0,9 & 0,3 & 0,2 \\
\hline $\begin{array}{c}10^{-4} \cdot D / r_{0}^{2} \\
\left(\mathrm{~s}^{-1}\right) \\
n \mathrm{C}_{5}\end{array}$ & & & & & \\
\hline
\end{tabular}

Après dissolution de la zéolithe dans l'acide fluorhydrique, une phase gazeuse et un extrait soluble dans le chlorure de méthylène ont été récupérés et analysés.

Jusqu'à $250^{\circ} \mathrm{C}$, seul le $n$-pentane est adsorbé dans les pores de la zéolithe 5A (tableau 3). Cet alcane est nécessairement piégé dans les pores de la zéolithe car il est trop volatil $\left(T_{\mathrm{eb}}=36,1^{\circ} \mathrm{C}\right)$ pour être adsorbé sur la surface externe de la zéolithe. À $420^{\circ} \mathrm{C}$, le $n$-pentane $(90 \%)$ est toujours le constituant majoritairement adsorbé dans les pores de la zéolithe mais, à côté, des molécules légères $\mathrm{C}_{2}$ à $\mathrm{C}_{6}$ sont détectées dans la phase gazeuse et des méthylbenzènes sont récupérés par extraction au chlorure de méthylène après solubilisation de la zéolithe dans l'acide fluorhydrique. Ces dernières molécules peuvent être dénommées coke et sont retenues dans les pores de la zéolithe à cause de leur taille.

À $100^{\circ} \mathrm{C}$, l'isopentane est le seul composé piégé dans les pores de la zéolithe (tableau 4). Comme dans le cas du $n$ pentane, il est trop volatil $\left(T_{\mathrm{eb}}=27,8^{\circ} \mathrm{C}\right)$ pour rester adsorbé sur la surface externe de la zéolithe. Quand la température augmente, l'isopentane reste le composé majoritaire de la phase adsorbée (tableau 4). Il est accompagné de composés légers plus ou moins insaturés $\left(\mathrm{C}_{2}\right.$ à $\left.\mathrm{C}_{6}\right)$, ainsi que de molécules plus volumineuses : oligomères (alcanes et alcènes) ayant de 15 à 24 atomes de carbone à $250^{\circ} \mathrm{C}$, et aromatiques substitués à $420^{\circ} \mathrm{C}$. Ces composés sont localisés et piégés dans les pores de la zéolithe à cause de leur faible volatilité (oligomères) et de leur taille (aromatiques).

\section{TABLEAU 3}

Composition de la phase adsorbée à partir du $n$-pentane

Composition of the adsorbed phase from n-pentane

\begin{tabular}{c|c|c|c}
\hline$T\left({ }^{\circ} \mathrm{C}\right)$ & 100 & 250 & 420 \\
\hline $\mathrm{m}(\%)$ & 13 & 9 & 4,5 \\
\hline Constituants $(\%)$ & $n \mathrm{C}_{5}(100 \%)$ & $n \mathrm{C}_{5}(100 \%)$ & $n \mathrm{C}_{5}(90 \%)$ \\
& & & $\mathrm{C}_{2} \rightarrow \mathrm{C}_{6}(5 \%)$ \\
& & & $0-\left(\mathrm{CH}_{3}\right)_{x}(5 \%)$ \\
& & & $x=2$ à 5 \\
\hline
\end{tabular}

TABLEAU 4

Composition de la phase adsorbée à partir de l'isopentane Composition of the adsorbed phase from isopentane

\begin{tabular}{c|c|c|c}
\hline$T\left({ }^{\circ} \mathrm{C}\right)$ & 100 & 250 & 420 \\
\hline $\mathrm{m}(\%)$ & 12 & 3 & 4,0 \\
\hline Constituants $(\%)$ & $i \mathrm{C}_{5}(100 \%)$ & $n \mathrm{C}_{5}(90 \%)$ & $i \mathrm{C}_{5}(85 \%)$ \\
& & $\mathrm{C}_{2} \rightarrow \mathrm{C}_{6}(5 \%)$ & $\mathrm{C}_{2} \rightarrow \mathrm{C}_{6}(5 \%)$ \\
& & Oligomères $(5 \%)$ & $\bigcirc-\left(\mathrm{CH}_{3}\right)_{x}$ \\
& & $\mathrm{C}_{15} \rightarrow \mathrm{C}_{24}$ & $x=2$ à $5(10 \%)$ \\
& & & ○ी $-\left(\mathrm{CH}_{3}\right)_{x}$ \\
& & & $x=1$ à 5 \\
\hline
\end{tabular}

\subsection{Désorption des composés adsorbés sur zéolithe 5A}

L'élimination des composés adsorbés (désorption) a été réalisée sous vide $(1 \mathrm{~Pa})$ à la température d'adsorption. La figure 5 montre sur l'exemple de l'isopentane les différences de vitesse de désorption pour 100 et $420^{\circ} \mathrm{C}$. À $100^{\circ} \mathrm{C}$, alors que la phase adsorbée est uniquement constituée d'isopentane, la désorption est très rapide et complète après 30 minutes. À $420^{\circ} \mathrm{C}$, la présence de composés aromatiques dans les pores de la zéolithe limite la désorption de l'isopentane, et après 1 heure de traitement, seulement $40 \%$ des composés adsorbés sont éliminables.

Quelles que soient les conditions opératoires considérées, les alcanes adsorbés à $100^{\circ} \mathrm{C}$ sont totalement éliminés des pores de la zéolithe après 1 heure de traitement sous vide. À $250^{\circ} \mathrm{C}$, le $n$-pentane adsorbé peut être facilement éliminé alors que seulement $80 \%$ de la phase adsorbée ex-isopentane est éliminable. Enfin, à $420^{\circ} \mathrm{C}$, si $80 \%$ des composés adsorbés à partir du $n$-pentane sont désorbés, seulement $40 \%$ le sont à partir de l'isopentane. Cette faible désorption peut être reliée à la présence de coke, qui limite la diffusion des molécules d'isopentane piégées dans des cavités proches de celles contenant les molécules de coke.

\section{DISCUSSION}

Malgré une diffusion plus faible de l'isopentane par rapport au $n$-pentane, l'analyse des composés adsorbés dans les pores de la zéolithe $5 \mathrm{~A}$ montre clairement que, quelle que soit la température, l'isopentane arrive à entrer dans les pores de la zéolithe et reste piégé dans les cages $\alpha$. Ce résultat semble surprenant puisque le diamètre cinétique de l'isopentane est apparemment plus grand que celui de l'entrée des pores de la zéolithe. Toutefois, nous avons montré que l'isopentane pouvait également pénétrer dans les pores d'une zéolithe $5 \mathrm{~A}$ moins échangée $(56 \%)$ et y rester bloqué [15]. De plus, il a également été montré que l'isobutène, l'isobutane [4] et, dans une moindre mesure, les méthylpentanes [15] pourraient eux aussi entrer dans les pores de la zéolithe 5A. 


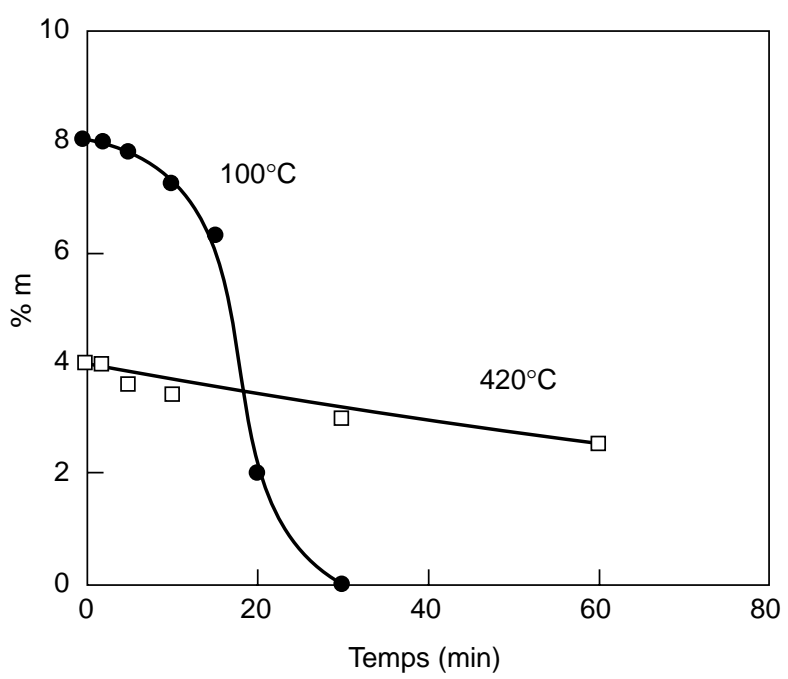

Figure 5

Désorption sous vide de la phase adsorbée sur zéolithe $5 \mathrm{~A}$ à partir de l'isopentane à 100 et $420^{\circ} \mathrm{C}$.

Desorption under vaccum of the adsorbed phase formed over $5 \mathrm{~A}$ zeolite from isopentane at 100 and $420^{\circ} \mathrm{C}$.

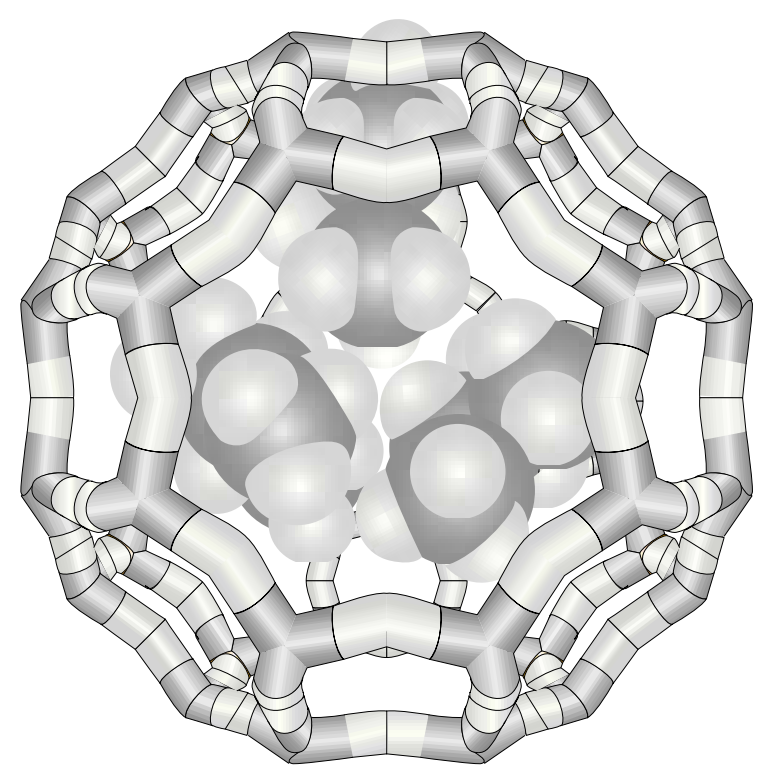

Figure 6

Modélisation moléculaire des molécules d'isopentane localisées dans la cage $\alpha$ de la zéolithe $5 \mathrm{~A}$.

Molecular modelling of isopentane molecules located in the $\alpha$ cage of 5 A zeolite.

\subsection{Localisation des molécules adsorbées}

À $50^{\circ} \mathrm{C}$, la prise de masse après 50 heures est égale à 12 et $13 \%$, respectivement pour l'iso- et le $n$-pentane, ce qui correspond à $10 \times 10^{20}$ et $11 \times 10^{20}$ molécules d'alcane adsorbées par gramme de zéolithe. Cette quantité étant environ trois fois plus importante que le nombre de cages $\alpha$, chaque cage $\alpha$ de la zéolithe 5A serait donc capable d'accueillir trois molécules de $n$-pentane ou d'isopentane, comme le montre la modélisation moléculaire à partir d'un logiciel CERIUS ${ }^{2}$ (fig. 6). En accord avec ceci, il a été montré que la zéolithe $5 \mathrm{~A}$ pouvait accueillir deux molécules de $n \mathrm{C}_{7}$ dans sa cavité [16] et cinq molécules de propane [17]. Toutefois, comme le montrent la figure 6 et le calcul, le volume occupé par trois molécules de pentanes $\left(\approx 580 \AA^{3}\right)$ est plus petit que le volume de la cage $\alpha\left(770 \AA^{3}\right.$ [18]), ce qui indique que la cage $\alpha$ n'est pas totalement remplie. Cependant, il semble exclu qu'une molécule supplémentaire de pentanes puisse être localisée dans la cage $\alpha$. Toutefois, à saturation à $50^{\circ} \mathrm{C}$, toutes les cages $\alpha$ de la zéolithe doivent être occupées par au moins trois molécules de pentanes.

À plus haute température, la quantité de $n$-pentane adsorbée à saturation est plus élevée que celle de l'isopentane. Jusqu'à $250^{\circ} \mathrm{C}$, cette différence ne peut être expliquée par une formation de coke à partir du $n$-pentane, mais plutôt par une diffusion plus facile et plus rapide de cet alcane dans les pores de la zéolithe par rapport à l'isopentane.

Ainsi, les molécules de $n$-pentane seraient distribuées de façon plus homogène dans les pores de la zéolithe que les molécules d'isopentane. Les molécules d'isopentane pourraient être préférentiellement adsorbées dans les cages les plus proches de la surface externe, limitant ainsi l'accès des autres molécules d'isopentane au centre de la cristallite.

En présence d'isopentane, à plus haute température $(T \geq$ $250^{\circ} \mathrm{C}$ ) la formation de molécules plus volumineuses (coke) entraîne une légère augmentation de la prise de masse (fig. 2). Ces molécules de coke doivent être localisées préférentiellement dans les cavités proches de la surface externe des cristallites. La présence de ces composés (coke), localisés dans les cavités les plus proches de la surface externe des cristallites de la zéolithe, limite certainement l'adsorption de l'isopentane mais aussi sa désorption, comme nous l'avons suggéré précédemment. En accord avec cela, sur un adsorbant industriel constitué de zéolithe $5 \mathrm{~A}$ et d'un liant, alors que l'adsorption de l'isopentane à $100^{\circ} \mathrm{C}$ n'est pas modifiée de façon significative [15], sa désorption est ralentie (fig. 7). La présence d'un liant, comme celle de molécules de coke localisées dans des cavités proches de la surface externe des cristallites de zéolithe, limite donc la désorption de l'isopentane des pores de la zéolithe 5A.

\subsection{Mode de transformation des molécules d'isopentane}

À haute température $\left(\mathrm{T} \geq 250^{\circ} \mathrm{C}\right)$, l'isopentane est transformé en composés légers : $\mathrm{C}_{2}-\mathrm{C}_{6}$ alcanes et alcènes, et en composés plus lourds (coke) : $\mathrm{C}_{15}-\mathrm{C}_{24}$ oligomères et $\mathrm{C}_{8}-\mathrm{C}_{15}$ aromatiques. 


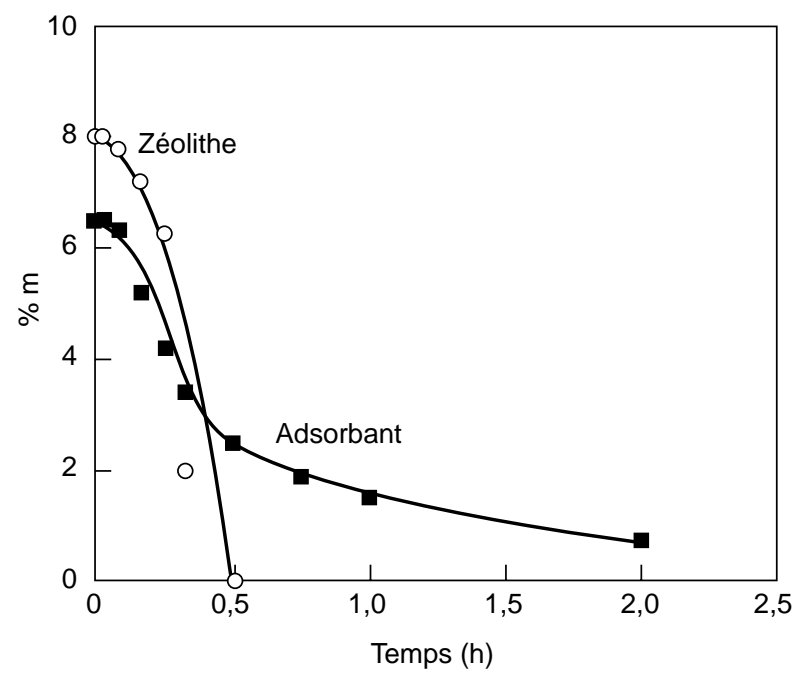

Figure 7

Désorption sous vide de 1 'isopentane piégé à $100^{\circ} \mathrm{C}$ sur la zéolithe $5 \mathrm{~A}$ et un adsorbant à base de zéolithe $5 \mathrm{~A}$.

Desorption under vaccum of isopentane trapped at $100^{\circ} \mathrm{C}$ over $5 A$ zeolite and $5 A$ adsorbent.

\subsubsection{Formation des composés légers}

La présence de sites acides sur la zéolithe $5 \mathrm{~A}$ et le temps de contact très long entre alcane et site acide permettent d'expliquer la formation des composés $\mathrm{C}_{2}-\mathrm{C}_{6}$, qui nécessite des réactions de craquage à partir d'ions carbéniums :

$$
\mathrm{C}-\underset{\mathrm{C}}{\mathrm{C}}-\mathrm{C}-\mathrm{C}^{+} \rightarrow \mathrm{C}-\stackrel{+}{\mathrm{C}}-\mathrm{C}+\mathrm{C}=\mathrm{C}
$$

Les alcanes branchés étant plus réactifs que les $n$-alcanes [19], le craquage de l'isopentane sera favorisé devant celui du $n$-pentane. De ce fait, la formation d'oléfines sera toujours plus importante avec l'isopentane, ainsi que la formation de coke.

Toutefois, l'absence de méthane et la présence de $\mathrm{C}_{4}$ et $\mathrm{C}_{6}$ ne peuvent être expliquées par la seule réaction de craquage. Il faut donc admettre qu'il existe une autre voie, telle que la réaction de dimérisation-craquage :

$$
\begin{aligned}
& \mathrm{C}-\underset{\mathrm{C}}{\mathrm{C}^{+}}-\mathrm{C}-\mathrm{C} \rightleftharpoons \mathrm{C}-\underset{\mathrm{C}}{\mathrm{C}}=\mathrm{C}-\mathrm{C} \\
& i \mathrm{C}_{5}{ }^{+} \quad i \mathrm{C}_{5}= \\
& i \mathrm{C}_{5}{ }^{+}+i \mathrm{C}_{5}=\mathrm{C}_{10}{ }_{\mathrm{C}_{4}{ }^{+}+\mathrm{C}_{6}{ }^{+}}
\end{aligned}
$$

\subsubsection{Formation des composés lourds (coke)}

Le coke est constitué d'oligomères (avec un nombre de carbones non multiple de 5) à $250^{\circ} \mathrm{C}$ et d'aromatiques à $420^{\circ} \mathrm{C}$. La formation de coke est toujours plus rapide avec l'isopentane qu'en présence de $n$-pentane (tableaux 3 et 4 ). Ceci est à relier à la plus grande réactivité de l'isopentane pour former des oléfines par craquage. Les alcènes résultant du craquage, ou de la réaction de dimérisation-craquage, peuvent réagir entre eux ou réagir avec un carbocation $i \mathrm{C}_{5}{ }^{+}$ pour conduire aux oligomères :

$$
\begin{array}{r}
i \mathrm{C}_{5}^{+} \rightarrow \text { oléfines + alcanes } \\
2 \text { oléfines } \stackrel{\mathrm{H}^{+}}{\rightarrow} \text { oligomères } \\
\text { oléfines }+i \mathrm{C}_{5}{ }^{+} \rightarrow \text { oligomères }
\end{array}
$$

Les composés aromatiques formés à haute température nécessitent quant à eux plusieurs réactions successives telles qu'oligomérisation, cyclisation (cycl), transfert d'hydrogène (TH), etc. :

$$
\text { oligomères } \stackrel{\text { cycl }}{\rightarrow} \text { cycloalcanes } \underset{\mathrm{TH}}{\rightarrow} \text { aromatiques }
$$

\section{CONCLUSION}

$\mathrm{Au}$ cours de l'adsorption de l'isopentane sur zéolithe 5A entre 50 et $420^{\circ} \mathrm{C}$, l'analyse de la phase adsorbée montre que malgré la taille et la faible diffusion de l'isopentane, ce dernier entre dans les pores de la zéolithe et reste piégé dans les cages $\alpha$.

À saturation à $50^{\circ} \mathrm{C}$, la prise de masse peut atteindre $12 \%$, les cages de la zéolithe étant totalement remplies par au moins trois molécules d'isopentane chacune. À cette température, ces molécules peuvent très facilement être désorbées par un traitement sous vide primaire.

Lorsque la température d'adsorption augmente, la répartition dans les pores de la zéolithe des molécules d'isopentane est beaucoup plus hétérogène et ces dernières sont certainement localisées dans les cavités proches de la surface externe. D'autre part, la présence de sites acides et la plus grande réactivité de l'alcane branché par rapport au $n$ alcane conduisent à la formation de composés légers $\mathrm{C}_{2}-\mathrm{C}_{6}$ et de molécules de coke (oligomères et composés mono- et biaromatiques). Ces derniers constituants sont retenus dans les cages $\alpha$ de la zéolithe à cause de leur faible volatilité (oligomères) et de leur taille (aromatiques), qui est intermédiaire entre celle de l'ouverture de la cage et l'espace disponible dans cette cavité. De plus, leur présence limite la désorption de l'isopentane des pores de la zéolithe. 


\section{RÉFÉREN CES}

1 Belloum, M., Travers, Ch. et Bournonville, J.P. (1991) Isomérisation des paraffines de $\mathrm{C}_{4}$ à $\mathrm{C}_{7}$ sur catalyseurs zéolithiques. Revue de l'Institut français du pétrole, 46, 1, 89-107.

2 Johnson, J.A. et Oroska, A.R. (1989) Sorbex Technology for Industrial Scale Separation. Stud. Surf. Sci. Catal., 46, 451467.

3 Jullian, S., Mank, L. et Minkkinen, A. (1993) Fr. Patent, 2. 679. 245, US Patent 5, 233, 120.

4 Misk, M., Joly, G., Magnoux, P., Jullian, S. et Guisnet, M. (1996) Coking, Aging and Regeneration of Zeolites: XVII. Composition and Location of Carbonaceous Compounds Resulting from Isobutene and Propene Transformation of a 5A Zeolite. Zeolites, 16, 265-270.

5 Thomazeau, C., Cartraud, P., Magnoux, P., Jullian, S. et Guisnet, M. (1996) Formation from Propene of Carbonaceous Compounds Responsible for the Deactivation of a $5 \mathrm{~A}$ Adsorbent. Removal through Pyrolysis and Oxidative Treatment. Microporous Materials, 5, 337-345.

6 Magnoux, P., Misk, M., Joly, G., Jullian, S. et Guisnet, M. (1997) Composition, Location, Modes of Formation and of Removal of Coke Deposited on a 5A Adsorbent. Stud. Surf. Sci. Catal., 105, 1835-1858.

7 Boucheffa, Y., Thomazeau, C., Cartraud, P., Magnoux, P., Guisnet, M. et Jullian, S. (1997) Formation of Carbonaceous Compounds from Propene and Isobutene over a 5A Zeolite. Influence of Temperature on their Compositions and Locations. Ind. Eng. Chem. Research, 36, 8, 3198-3204.

8 Freude, D., Kärger, J. et Pfeifer, H. (1985) Lattice Defects and Surface Barriers in Thermally Treated Zeolite CaA. Proceedings of the International Symposium on Zeolite Catalysis, Siofok, 89-98.

9 Mix, H., Pfeifer, H. et Staudte, B. (1988) Hydroxyl Groups in Zeolites CaNaA. Chem. Phys. Lett., 146, 6, 541-544.
10 Guisnet, M. et Magnoux, P. (1989) Coking and Deactivation of Zeolites. Influence of the Pore Structure. Appl. Catal., 54, $1-27$.

11 Magnoux, P., Roger, P., Canaff, C., Fouché, V., Gnep, N.S. et Guisnet, M. (1987) New Technique for the Characterization of Carbonaceous Compounds Responsible for Zeolite Deactivation. Stud. Surf. Sci. Catal., 34, 317-330.

12 Bin, L., Shaozhou, C., Xiaolong, Z. et Chao, T. (1995) Adsorptive Separation of Light Normal and Iso-Paraffins on 5A Molecular Sieve. Acta Petrolei Sinica, 3, 40-45.

13 Silva, J.A.C. et Rodrigues, A.E. (1997) Sorption and Diffusion of $n$-Pentane in Pellets of 5A Zeolite. Ind. Eng. Chem. Res., 36, 493-500.

14 Goddard, M. et Ruthen, D.M. (1986) Sorption and Diffusion of $\mathrm{C}_{8}$ Aromatic Hydrocarbons in Faujasite Type Zeolites. II. Sorption Kinetics and Intracrystalline Diffusivities. Zeolites, 6, 283-289.

15 Boucheffa, Y. (1997) Adsorption d'oléfines et de paraffines sur la zéolithe 5A. Thèse, Poitiers.

16 Doetsch, I.H., Ruthven, D.M. et Loughlin, K.F. (1974) Sorption and Diffusion of $n$-Heptane in 5A Zeolite. Can. J. Chem., 52, 2717-2725.

17 Ruthven, D.M. (1984) in Principles of Adsorption and Adsorption Process, John Wiley and Sons, New York.

18 Breck, D.W. (1974) in Zeolite Molecular Sieves, John Wiley and Sons, New York.

19 Guisnet, M.R. (1990) Model Reactions for Characterizing the Acidity of Solid Catalysts. Acc. Chem. Res., 23, 11, 392-398.

Manuscrit définitif reçu en décembre 1999 